\title{
MEASUREMENT, EVALUATION AND VALIDATION OF MOLYBDENUM CROSS SECTIONS
}

\author{
Isabelle Duhamel $^{1}$, Nicolas Leclaire ${ }^{1}$, Luiz Leal ${ }^{1}$, Atsushi Kimura ${ }^{2}$, Shoji Nakamura ${ }^{2}$ \\ ${ }^{1}$ Institut de Radioprotection et de Sûreté Nucléaire (IRSN) \\ Fontenay-aux-Roses, 92262, France \\ 2 Japan Atomic Energy Agency (JAEA), Tokai, 319-1195, Japan \\ isabelle.duhamel@irsn.fr, nicolas.leclaire@irsn.fr, luiz.leal@irsn.fr, \\ kimura.atsushi04@jaea.go.jp, nakamura.shoji@jaea.go.jp
}

\begin{abstract}
Available nuclear data for molybdenum included in the nuclear data libraries are not of sufficient quality for reactor physics or criticality safety issues and indeed information about uncertainties and covariance is either missing or leaves much to be desired. Therefore, IRSN and JAEA performed experimental measurements on molybdenum at the J-PARC (Japan Proton Accelerator Research Complex) facility in Japan. The aim was to measure capture cross section and transmission of natural molybdenum at the ANNRI (Accurate NeutronNucleus Reaction measurement Instrument) in the MLF (Material Life and science Facility) of J-PARC. The measurements were performed on metallic natural molybdenum samples with various thicknesses. A NaI detector, placed at a flight-path length of about $28 \mathrm{~m}$, was used for capture measurements and a Li-glass detector (flight-path length of about $28.7 \mathrm{~m}$ ) for transmission measurements. Following the data reduction process, the measured data are being analyzed and evaluated to produce more accurate cross sections and associated uncertainties.
\end{abstract}

KEYWORDS: differential experiments, molybdenum, capture, transmission, J-PARC

\section{INTRODUCTION}

A precise knowledge of neutron cross sections is of a great importance to accurately calculate reaction rates and detailed neutron flux distributions in many nuclear applications. Reducing uncertainties in the neutron cross section data can result in an enhanced safety of present and future nuclear systems.

Molybdenum isotopes are mainly encountered in irradiated fuel as fission products or in molybdenum alloys in research or naval reactors. In the nuclear fuel cycle, ${ }^{95} \mathrm{Mo}$ is also taken into account in criticality safety studies considering burn-up credit for transportation casks or irradiated fuel storage. ${ }^{95} \mathrm{Mo}$ is one of the 15 main absorbing fission products in Light Water Reactors (LWR) irradiated fuel assemblies. Besides, in reprocessing plants, during the dissolution process, some UPu-MoZr deposits could appear in specific equipments. Thus, accurate nuclear data of molybdenum isotopes in a wide energy range are of great importance. As an example, molybdenum isotopes are among the greatest contributors to the global nuclear data uncertainty on calculated $\mathrm{k}_{\text {eff }}$ in $\mathrm{UPu}-\mathrm{MoZr}$ deposits configurations.

The available nuclear data for molybdenum included in the nuclear data libraries are not of sufficient quality for reactor physics or criticality safety issues and indeed information about uncertainties and 
covariance are either missing or leaves much to be desired. Therefore, IRSN and JAEA performed experimental measurements on molybdenum at the J-PARC (Japan Proton Accelerator Research Complex) facility in Japan. The aim was to measure the capture cross section and transmission of natural molybdenum.

\section{MEASUREMENTS AT THE J-PARC FACILITY}

In 2018, IRSN submitted a proposal to the J-PARC system to perform experiments with molybdenum samples and was granted 7 days of neutron beam. Molybdenum capture cross sections and transmission measurements were performed on five metallic natural molybdenum samples of various thicknesses. Additional measurements were performed to determine the background and normalization factors.

\subsection{J-PARC facility}

J-PARC is a proton accelerator facility operated by JAEA and KEK in Tokai-Mura. The measurements were carried out using the ANNRI (Accurate Neutron-Nucleus Reaction measurement Instrument) in the MLF (Material Life and science Facility for the purpose of using neutrons to investigate material properties) of J-PARC [1].

Neutrons were produced via spallation reactions induced by a $3-\mathrm{GeV}$ pulsed proton beam impinging on a mercury target of MLF. The accelerator was operated in single-bunch mode at a repetition rate of $25 \mathrm{~Hz}$. The proton beam power on the spallation target was around $500 \mathrm{~kW}$. The number of incident protons was recorded and used for normalization of each measurement.

3 types of detectors are available (see Figure 1) at the ANNRI, allowing for transmission and capture cross sections measurements:

- a $4 \pi$ germanium spectrometer,

- a NaI spectrometer,

- a Li-glass detector.

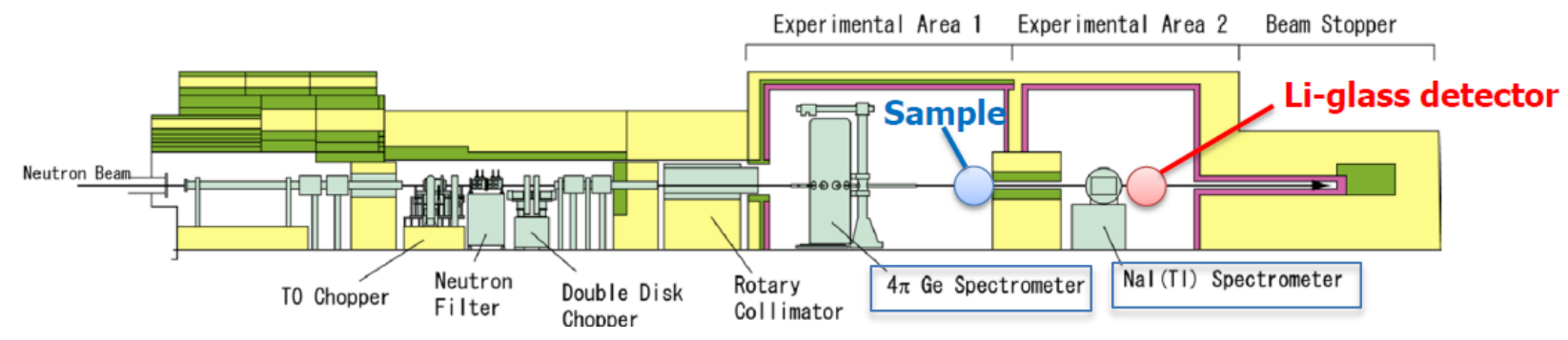

Figure 1. ANNRI experimental device

(The sample position shown corresponds to transmission measurements using Li-Glass detector)

\subsection{Capture cross-section measurements}

A NaI detector (flight-path length of about $28 \mathrm{~m}$ ) was used for capture measurements (see Figure 2). 

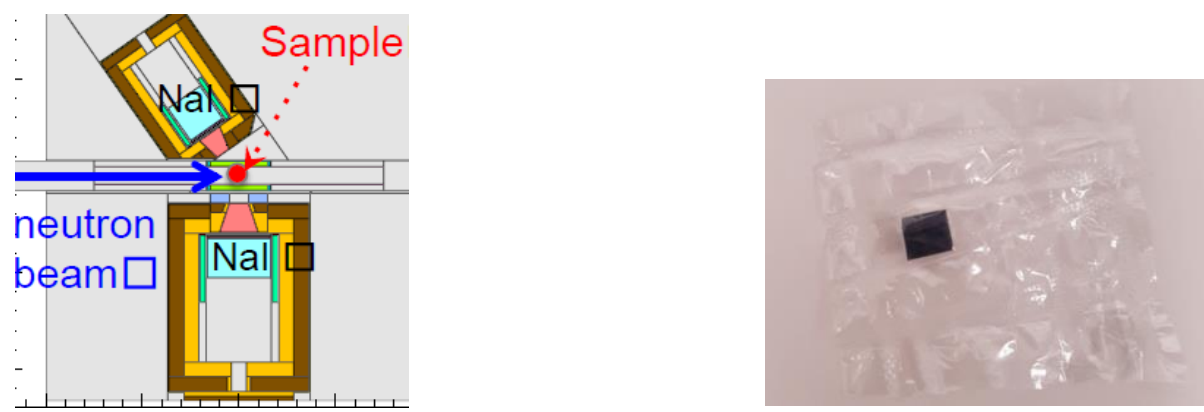

Figure 2. NaI detector in ANNRI (left) and $0.1 \mathrm{~mm}$ Mo sample in the FEP film (right)

Three samples of various thicknesses were used during the measurements: $0.1 \mathrm{~mm}, 0.5 \mathrm{~mm}$ and $2 \mathrm{~mm}$ made by the stacking of four $0.5 \mathrm{~mm}$ samples. Caution was taken to ensure that there is no air gap between the 4 samples and reproducibility experiment was performed. The samples weight was measured using a micro analytical balance AND BM 22 type, which precision is $0.001 \mathrm{mg}$. Table I gives the characteristics of the Mo samples used for capture measurements.

Table I. Characteristics of the Mo samples for capture measurements

\begin{tabular}{|c|c|c|}
\hline Thickness (mm) & Dimensions (mm x mm) & Weight (mg) \\
\hline 0.1 & $4.9 \times 4.4^{\mathrm{a}}$ & 21.025 \\
\hline 0.5 & $5 \times 5^{\mathrm{c}}$ & 128.023 \\
\hline $2^{\mathrm{b}}$ & $5 \times 5^{\mathrm{c}}$ & 541.747 \\
\hline
\end{tabular}

a. Dimensions were measured using a micrometer, which precision is $0.05 \mathrm{~mm}$

b. The sample was made by stacking four $0.5 \mathrm{~mm}$ thick samples.

c. Cutting specifications

Additional reference measurements were also performed on lead, gold and boron in order to determine the normalization factors for the capture cross section. The lead sample is a Pb-208 sample, which is $5 \mathrm{~mm}$ in diameter and $159.7 \mathrm{mg}$ in weight. The gold reference sample is a $6 \mathrm{~mm}$ diameter Au-197 sample with a thickness of $10 \mu \mathrm{m}$.

Blank measurements, meaning only with the FEP film used to cover the samples, were also performed to determine the background of capture measurements.

\subsection{Transmission measurements}

Transmission measurements were performed on a thin metallic sample $(0.5 \mathrm{~mm})$ and a thick one $(5 \mathrm{~mm})$ using a Li-glass detector (flight-path length of $28.71 \mathrm{~m}$ ). Table II gives the characteristics of the Mo samples used for transmission measurements. Figure 1 shows the sample and Li-glass detectors position. Figure 3 shows the Li-glass detectors and the $5 \mathrm{~mm}$ thick Mo sample installed on the experimental device used for the transmission measurements.

Table II. Characteristics of the Mo samples for transmission measurements

\begin{tabular}{|c|c|c|c|}
\hline Thickness $(\mathrm{mm})$ & \multirow{2}{*}{ Bin width $(\mu \mathrm{s})$} & $\begin{array}{c}\text { Surface density } \\
\left(\mathrm{g} / \mathrm{cm}^{2}\right)\end{array}$ & $\begin{array}{c}\text { Sample thickness } \\
(\text { atoms } / \text { barn })\end{array}$ \\
\hline \multirow{2nny}{*}{0.5} & \multirow{2}{*}{1} & 0.538 & $3.37664 \mathrm{E}-03$ \\
\cline { 3 - 4 } 5 & \multirow{2}{*}{0.1} & 5.091 & $3.19526 \mathrm{E}-02$ \\
\hline 0.5 & & 0.538 & $3.37664 \mathrm{E}-03$ \\
\cline { 3 - 4 } 5 & & 5.091 & $3.19526 \mathrm{E}-02$ \\
\hline
\end{tabular}



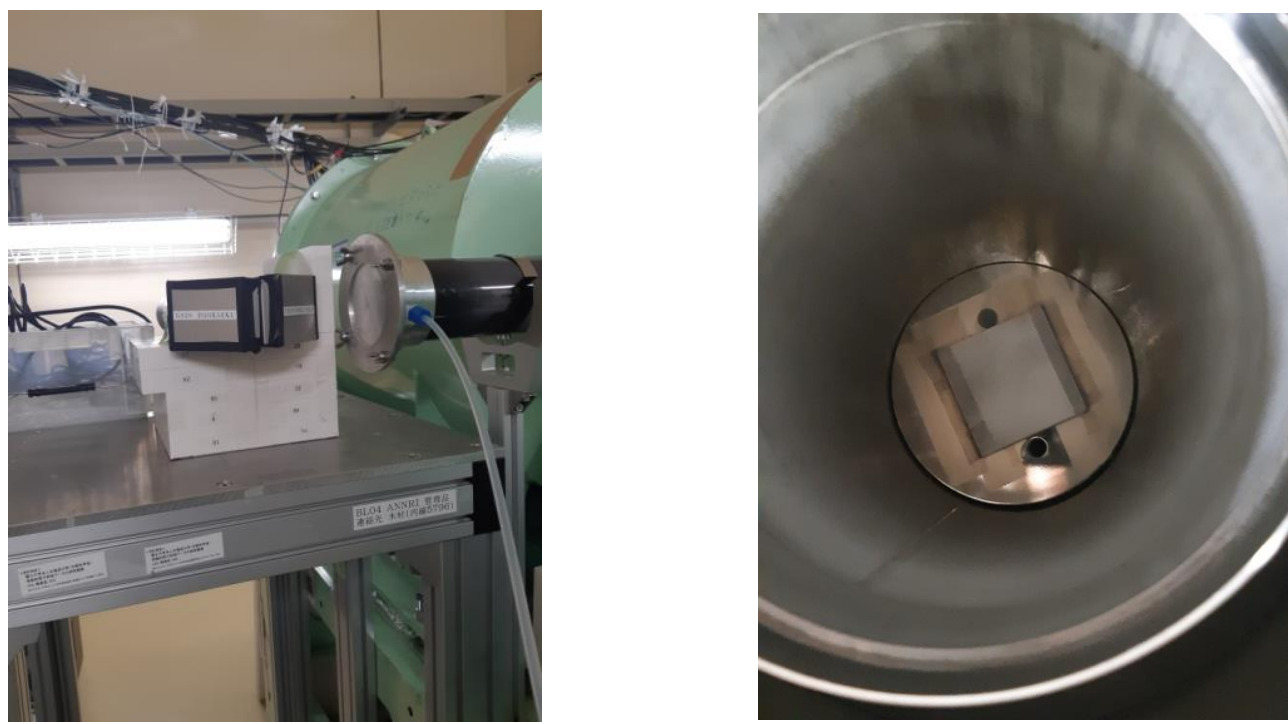

Figure 3. Li-glass detectors (left) and $5 \mathrm{~mm}$ thick Mo sample (right) installed on the experimental device used for the transmission measurements

Additional measurements were performed using $\mathrm{Co}, \mathrm{Mn}, \mathrm{In}, \mathrm{Ag}$ resonance filters. Blank measurement (without sample) was also performed to check the Background.

\section{MEASUREMENTS ANALYSIS AND EVALUATION}

Natural molybdenum on which the transmission measurements were performed is comprised of seven major isotopes, amongst which two are particularly important for criticality safety, namely, ${ }^{95} \mathrm{Mo}$ and ${ }^{96}$ Mo since they have strong resonances in the low energy range (see Figure 4).

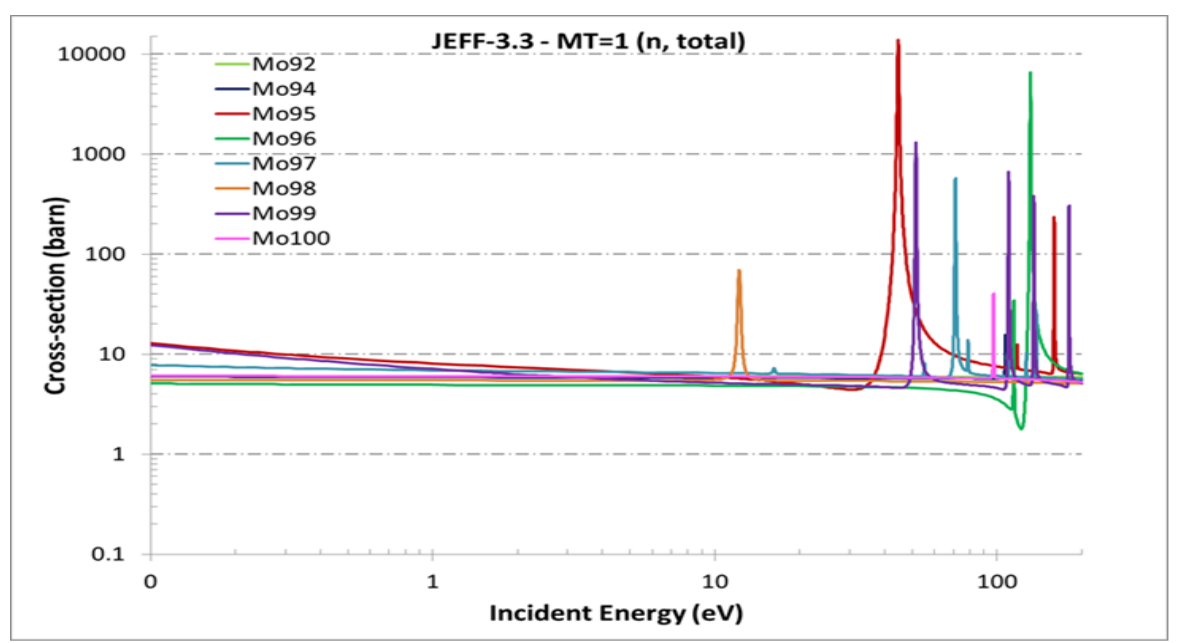

Figure 4. Mo isotopes total cross sections.

The abundances of molybdenum stable isotopes and associated thermal cross sections and resonance integral are reported in Table III. The target spin is $0^{+}$for ${ }^{92} \mathrm{Mo},{ }^{94} \mathrm{Mo},{ }^{96} \mathrm{Mo},{ }^{98} \mathrm{Mo},{ }^{100} \mathrm{Mo}$ and $5 / 2$ for 
${ }^{95} \mathrm{Mo},{ }^{97} \mathrm{Mo}$. An inspection of the evaluations included in the ENDF (JEFF-3.3 evaluation and others) show that resonance parameters for ${ }^{95} \mathrm{Mo}$ are available from $10^{-5} \mathrm{eV}$ to $2145 \mathrm{eV}$ with 23 resonances corresponding to $l=0$ (s-wave) and 34 resonances to $l=1$ ( $p$-wave). However, in the JEFF-3.3 evaluation [2], as well as ENDF, the correspondence between the spin groups and the channel spin is not correctly addressed for these odd isotopes. Work has been done to correct this issue and assign physically accepted spin sequences.

Table III. Abundances of molybdenum isotopes and associated thermal cross sections and resonance integrals.

\begin{tabular}{|c|c|c|c|}
\hline Isotope & Abundance (atom \%) & $\begin{array}{c}\text { Thermal Cross } \\
\text { Section (barns)* }\end{array}$ & $\begin{array}{c}\text { Resonance Integral } \\
\text { (barns)* }\end{array}$ \\
\hline${ }^{92} \mathrm{Mo}$ & 14.84 & $0.08 \pm 0.02$ & 0.83 \\
\hline${ }^{94} \mathrm{Mo}$ & 9.25 & $0.34 \pm 0.02$ & 1.12 \\
\hline${ }^{95} \mathrm{Mo}$ & 15.92 & $13.4 \pm 0.3$ & $118 \pm 7$ \\
\hline${ }^{96} \mathrm{Mo}$ & 16.68 & $0.5 \pm 0.3$ & $17 \pm 3$ \\
\hline${ }^{97} \mathrm{Mo}$ & 9.55 & $2.2 \pm 0.2$ & $14.4 \pm 3.0$ \\
\hline${ }^{98} \mathrm{Mo}$ & 24.13 & $0.130 \pm 0.006$ & $6.7 \pm 0.3$ \\
\hline${ }^{100} \mathrm{Mo}$ & 9.63 & $0.199 \pm 0.002$ & $3.76 \pm 0.15$ \\
\hline
\end{tabular}

* Mughabghab

\subsection{Channel spin for the compound nucleus $n+\operatorname{target}$ for ${ }^{95} \mathrm{Mo}$ and ${ }^{97} \mathrm{Mo}$}

The channel spin $s$ in the entrance channel is determined by a combination of the neutron spin $(1 / 2)$ and the target spin $I$. Hence, according to the quantum spin addition rule the entrance channel spin can be $s=1 / 2$ - $I$ and $\mathrm{s}=1 / 2+I$. It is easy to see that for target spin $I$ greater than 0 there will always be two possible channel spin. The resonance total angular momentum $j$ is obtained by combination of the spin channel $s$ and orbital angular momentum $l$. The isotopes of molybdenum ${ }^{95} \mathrm{Mo}$ and ${ }^{97} \mathrm{Mo}$ have spin of $5 / 2$. Existing evaluations for these isotopes (ENDF, JEFF, JENDL, etc) do not consider separation of the two spin channels for $l>0$. Consequently, interference effects in the scattering cross section (total cross section) are not properly accounted for. The evaluation presented in this work takes into account the spin channel issue in the fitting procedure by the SAMMY code [3]. It is of great importance since it can reflect the interferences in the entrance channels.

The quantum numbers $j, l$, and $s$ are provided in Table IV for ${ }^{95} \mathrm{Mo}$ and ${ }^{97} \mathrm{Mo}$. The neutron spin and parity is $1 / 2+$.

Table IV. Channel spins for molybdenum isotopes.

\begin{tabular}{|c|c|c|c|}
\hline Isotope $(\mathrm{I}=5 / 2+)$ & $l$ & $s(1 / 2+I)$ & $j^{\pi}$ \\
\hline \multirow{8}{*}{${ }^{95} \mathrm{Mo},{ }^{97} \mathrm{Mo}$} & \multirow{2}{*}{0} & 2 & $2+$ \\
\hline & & 3 & $3+$ \\
\hline & \multirow{6}{*}{1} & 2 & $1-$ \\
\hline & & 2 & \multirow{2}{*}{$2-$} \\
\hline & & 3 & \\
\hline & & 2 & \multirow{2}{*}{ 3- } \\
\hline & & 3 & \\
\hline & & 3 & $4-$ \\
\hline
\end{tabular}




\subsection{SAMMY fit of the experimental data}

In the sequential fit of the experimental data at each step of the evaluation process an updated resonance parameter and covariance is generated, which are, subsequently feedback into the analysis of the next experimental data in the sequence. This process is repeated several times until a good fit of the experimental data is achieved. An important figure-of-merit that indicates a good fitting of the experimental data is the $\chi^{2}$ provided at the end of each SAMMY run. The resulting set of resonance parameters is expected to reproduce well the experimental data within the quoted experimental uncertainty.

Using the transmission measurements performed at the J-PARC facility for natural molybdenum, IRSN began a SAMMY fit of the $0.5-\mathrm{mm}$ thickness sample in the energy range $0-600 \mathrm{eV}$. The covariance matrix of cross sections was produced as an output. The resonance parameter and covariance were used for fitting the experimental data corresponding to the 5-mm thickness sample. The results of the SAMMY fitting of the experimental data are shown in Figure 5. The data resolution above $350 \mathrm{eV}$ is not good enough to obtain a good fit of the experimental data.
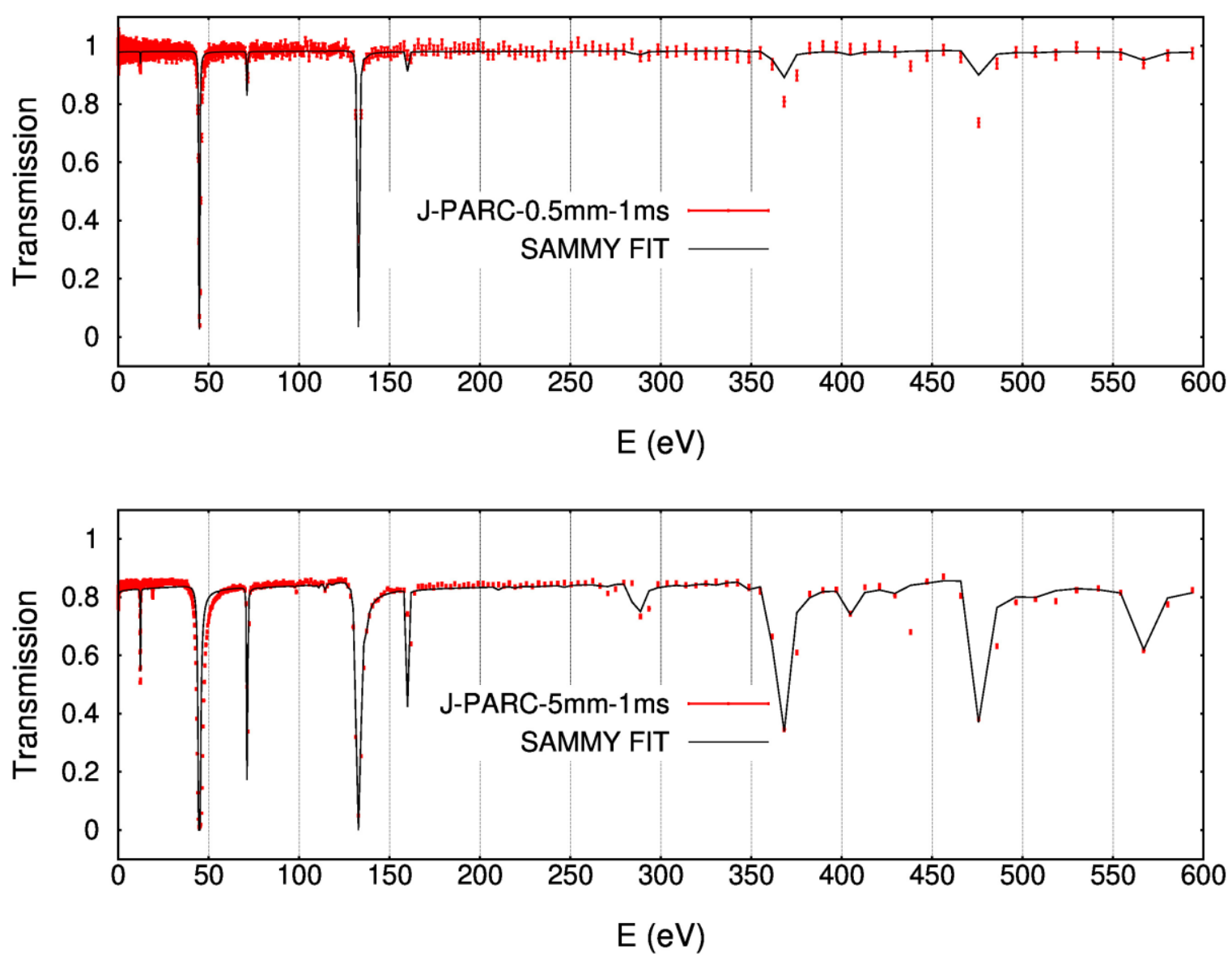

Figure 5. Fit of the J-PARC measurement values for $0.5-\mathrm{mm}$ (top) thick sample and reconstruction of resonance for 5-mm (bottom) thick sample. 


\subsection{Comparison of thermal cross section values}

Comparisons of the thermal capture and scattering cross sections $(0.0253 \mathrm{eV})$ between SAMMY results, calculated with resonance parameters derived in this work, based on the J-PARC measurements and reference values from Mughabghab [4] were done for ${ }^{95} \mathrm{Mo},{ }^{96} \mathrm{Mo}$ and ${ }^{97} \mathrm{Mo}$ isotopes. They are reported in Table V. Good agreement is observed between the calculated value and the reference one, giving quite good confidence in the produced fit.

Table V. Comparison of thermal cross sections at $0.0253 \mathrm{eV}$.

\begin{tabular}{|c|c|c|c|c|}
\hline \multirow{2}{*}{ Isotope } & \multicolumn{2}{|c|}{ SAMMY } & \multicolumn{2}{c|}{ Mughabghab } \\
\cline { 2 - 5 } & $\sigma_{\gamma}$ (barns) & $\sigma_{\mathrm{s}}$ (barns) & $\sigma_{\gamma}$ (barns) & $\sigma_{\mathrm{s}}$ (barns) \\
\hline${ }^{95} \mathrm{Mo}$ & 13.635 & 6.2112 & $13.4 \pm 0.3$ & $6.49 \pm 0.37$ \\
\hline${ }^{96} \mathrm{Mo}$ & 0.439 & 5.06575 & $0.5 \pm 0.2$ & $4.85 \pm 0.09$ \\
\hline${ }^{97} \mathrm{Mo}$ & 2.55741 & 6.64238 & $2.2 \pm 0.2$ & $6.61 \pm 0.14$ \\
\hline
\end{tabular}

\section{FUTURE WORK AND PERSPECTIVES}

Capture cross sections measurements were also performed at the J-PARC facility and the data reduction process is in progress. A SAMMY fitting will be performed on these measurements in order to provide more accurate capture data for molybdenum isotopes.

Besides, additional measurements on ${ }^{95} \mathrm{Mo},{ }^{96} \mathrm{Mo}$, and ${ }^{97} \mathrm{Mo}$ enriched samples are already envisioned for the next years.

The SAMMY fitting procedure has currently been applied to the J-PARC measured data in the 0-600 eV energy range. Future works will consist of extending the evaluation to higher energies covering at least the range of resonance parameters up to $2145 \mathrm{eV}$. It is expected that this range could be extended beyond this energy to account for resonances identified in the measured data.

The production of covariance data for transmission and capture cross sections for molybdenum is essential for criticality safety applications. Indeed, this data is used in the GLLSM methodology to establish the prior uncertainty due to nuclear data in criticality safety applications, which is the result of the propagation in terms of $\Delta \mathrm{k}_{\text {eff }}$ of cross sections uncertainties from the covariance matrix. Molybdenum isotopes covariances will be established using these new J-PARC measurements, which will surely help criticality practitioners with establishment of the bias applied on their industrial configurations using tools such as MACSENS [5].

Finally, the impact of the new molybdenum isotopes evaluation on benchmark calculation will be studied, on a basis of about 30 critical experiments issued from the ICSBEP handbook [6] and French proprietary programs [7] involving natural molybdenum in various neutron energy spectra. Moreover, new integral experiments involving molybdenum in thermal spectra are being designed to be performed at SPRF/CX at the SANDIA National Laboratories [8]. These future experiments will also be an opportunity to validate the new molybdenum nuclear data produced in the frame of this collaborative work between JAEA and IRSN. 


\section{CONCLUSIONS}

New differential experiments were performed at J-PARC facility on natural molybdenum samples of various thicknesses in the frame of a JAEA-IRSN collaboration. The transmission measurements were analyzed and the SAMMY fitting procedure was already applied on the $0-600 \mathrm{eV}$ energy range. On-going work is focused on the capture experimental data analysis and on the extension of the evaluation to higher energies covering at least the range of resonance parameters up to $2145 \mathrm{eV}$. Resonance parameter covariances for the evaluated isotopes will also be derived. The impact of the new molybdenum isotopes evaluation on benchmark calculation will be studied using integral experiments involving natural molybdenum in various neutron energy spectra.

This collaborative work between IRSN and JAEA will help to address issues concerned to criticality safety and reactor physics applications thru more accurate data and uncertainties.

\section{ACKNOWLEDGMENTS}

The neutron experiment at the Materials and Life Science Experimental Facility of the J-PARC was performed under a user program (Proposal No. 2018P0101).

\section{REFERENCES}

1. Y. Kiyanagi, "Accurate Neutron Nucleus Reaction Measurement Instrument (ANNRI) for Capture Cross Section Measurements at J-PARC", Journal of the Korean Physical Society, Vol. 59, No. 2, , pp. $779-784(2011)$

2. A. Plompen \& al, "The joint Evaluated Fission and Fusion Nuclear Data Library, JEFF-3.3", European Physical Journal, to be published

3. N. M. LARSON, "Updated Users' Guide for SAMMY: Multilevel R-Matrix Fits to Neutron Data Using Bayes' Equations," ORNL/TM-9179/R8 (ENDF-364/R2) (2008).

4 S. F. Mughabghab, "Atlas of Neutron Resonances - Resonance Parameters and Thermal Cross Sections Z $=1-100$," 5th Edition, National Nuclear Data Center, Brookhaven National Laboratory, Upton, USA (2006).

5 F. Fernex, Y. Richet, Y. Deville, "MACSENS: a software workbench for criticality calculation validation", Proc. ICNC2011, Edinburgh.

6 ICSBEP International Handbook of Evaluated Criticality Safety Benchmark Experiments, Organization of Economic Cooperation and Development-Nuclear Energy NEA/NSC/DOC(95)03, September 2016 Edition

7 N. Leclaire, I. Duhamel, F.X. Le Dauphin, J. Piot, J.B. Briggs, M. Rennesson, A. Laville, The MIRTE experimental program: an opportunity to test structural materials in various configurations in thermal energy spectrum, Nucl. Sci. Eng., (December 2014).

8 N. Leclaire, I. Duhamel, M. Monestier, "Use of advanced optimization algorithms for the design of critical experiments in support to criticality-safety assessment”, Proc. NCSD2017, Carlsbad ,USA 